\title{
Membrane-bound complement regulatory proteins are prognostic factors of operable breast cancer treated with adjuvant trastuzumab: A retrospective study
}

\author{
MEI LIU ${ }^{1,3}$, YA-JUN YANG ${ }^{1,2}$, HONG ZHENG $^{1,2}$, XIAO-RONG ZHONG ${ }^{1,2}$, YU WANG $^{1,2}$, \\ ZHU WANG $^{1,2}$, YAO-GENG WANG ${ }^{1,2}$ and YAN-PING WANG ${ }^{1,2}$ \\ ${ }^{1}$ Laboratory of Tumor Molecular Diagnosis and ${ }^{2}$ State Key Laboratory of Biotherapy, West China Hospital, \\ Sichuan University, Chengdu, Sichuan 610041; ${ }^{3}$ Chongqing Cancer Institute, Chongqing 400030, P.R. China
}

Received June 25, 2014; Accepted August 20, 2014

DOI: $10.3892 /$ or.2014.3496

\begin{abstract}
Complement-dependent cytotoxicity (CDC) is an important antitumor mechanism of monoclonal antibodies (mAbs). However, trastuzumab, an anti-HER $2 \mathrm{mAb}$, exerts only minor CDC. Overexpression of membrane-bound complement regulatory proteins (mCRPs), which suppress $\mathrm{CDC}$, have been implicated in various malignant tumors. Here, we explored the predictive role of the expression levels of three mCRPs (CD55, CD59 and CD46) in the prognosis of breast cancer cases that underwent adjuvant trastuzumab treatment. We also studied the effect of mCRP downregulation on trastuzumab-induced CDC in vitro. Sixty-five HER2positive breast cancer patients who received adjuvant therapy containing trastuzumab, were retrospectively analyzed. Levels of CD55, CD59 and CD46 expression were detected by immunohistochemistry. Chi-square test, Kaplan-Meier survival analysis and a Cox proportional hazards model were used to analyze the association between CD55, CD59 and CD46 expression and prognosis. HER2-positive SK-Br3 and BT-474 breast cancer cells were pretreated with various drugs to reduce $\mathrm{mCRP}$ expression. Afterwards, trastuzumab-mediated cytolytic effects were measured. Among the 65 patients, $46.2 \%$ had high expression of CD55, 44.6\% had high expression of CD59 and $44.6 \%$ had high expression of CD46. The median follow-up duration was 47 months (range from 24 to 75 months). Patients with CD55 or CD59 overexpression had a higher relapse rate than those with low expression of CD55 (33.3 vs. 8.6\%; $\mathrm{P}=0.013$ ) or CD59 (31.0 vs. $11.1 \%$; $\mathrm{P}=0.046$ ).
\end{abstract}

Correspondence to: Professor Yan-Ping Wang, Laboratory of Tumor Molecular Diagnosis and State Key Laboratory of Biotherapy, West China Hospital, Sichuan University, Chengdu, Sichuan 610041, P.R. China

E-mail: wyanping@scu.edu.cn

Key words: breast cancer, trastuzumab, complement-dependent cytotoxicity, membrane-bound complement regulatory proteins, 4-hydroxy tamoxifen
Similarly, mean disease-free survival of patients with CD55 or CD59 overexpression was significantly shorter than those with a low expression of CD55 (56 vs. 70 months; log-rank test, $\mathrm{P}=0.008$ ) or CD59 (56 vs. 69 months; log-rank test, $\mathrm{P}=0.033$ ). Multivariate analysis confirmed that CD55, but not CD59, was an independent risk factor of recurrence $(\mathrm{HR}=4.757 ; 95 \% \mathrm{CI}$, $0.985-22.974 ; \mathrm{P}=0.05)$. In vitro, we found that tamoxifen inhibited both the protein and mRNA expression levels of CD55, but not CD59 or CD46 in SK-Br3 and BT-474 cells. After pretreatment of tamoxifen, trastuzumab-induced cytolysis was enhanced through CD55 downregulation. In conclusion, CD55 overexpression is an independent risk factor for recurrence in breast cancer patients receiving postoperative adjuvant therapy containing trastuzumab. Combined use of tamoxifen and trastuzumab for HER2-positive breast cancer treatment may enhance the antitumor effects of trastuzumab by elevated CDC, which warrants further study.

\section{Introduction}

In recent years, monoclonal antibodies (mAbs) have been widely used in the treatment of malignant tumors including breast cancer, lymphoma and colorectal cancer $(1,2)$. The HER2-targeted humanized mAb trastuzumab has been recommended for patients with HER2-positive early and metastatic breast cancer. Compared with conventional chemotherapy, trastuzumab can significantly improve disease-free survival (DFS) and overall survival (OS) of patients (3-5). However, 30-50\% of HER2-positive breast cancer patients are not sensitive to trastuzumab. The mechanism of trastuzumab resistance has not been fully elucidated. There are few biological predictors of the efficacy of trastuzumab apart from HER2 (6,7).

Antibody-dependent cell-mediated cytotoxicity (ADCC) and complement-dependent cytotoxicity (CDC) are important immune mechanisms of the antitumor effects of mAbs, in addition to suppression of the HER2 signaling pathway, tumor cell apoptosis and inhibition of angiogenesis (6-8). Several IgG1 mAbs (e.g. rituximab, alemtuzumab and cetuximab) have been approved for the treatment of malignant tumors. Their antitumor effect via CDC has been clearly demonstrated in 
in vitro studies (9-12). Trastuzumab is a humanized IgG1 antibody. It binds to $\mathrm{Fc}$ receptors (FcRs) on immune effector cells or complements through its $\mathrm{Fc}$ fragment and induces ADCC or CDC. However, many studies have shown that trastuzumab induces minor or even no CDC $(9-12,13)$.

Activation of the complement system is an important mechanism by which to kill tumor cells. The complement system can be activated by antigen-antibody complexes via the classical pathway and by tumor cells via the alternative pathway. Ultimately, C5b, C6, C7, C8 and C9 bind together to form the membrane attack complex (MAC). The MAC attaches to the surface of tumor cells, leading to the lysis of cells, which is the process of CDC (8). Membrane-bound complement regulatory proteins (mCRPs) can hinder complement pathway activation at different points and thus reduces CDC. mCRPs protect normal cells from complement-mediated cytolytic effects. Therefore, it is conceivable that this may be the mechanism of the tolerance of tumor cells to mAb-induced CDC. CD55, CD59 and CD46 are the most extensively studied mCRPs in tumor cells (14-16). Overexpression of one or more mCRPs has been found in different types of cancers, including colorectal, lung, prostate and breast cancer (17-21). Highly expressed mCRPs may block the complement pathway and thus protect tumor cells from mAb-induced CDC. Terui et al (22) detected CD55 and CD20 expression on the surface of B lymphoma tumor cells and observed the CDC effect induced by rituximab. They found that CD55 overexpression decreased the susceptibility to rituximab-induced CDC of B lymphoma tumor cells. Golay et al (23) reported that downregulation of CD55 and CD59 enhanced rituximab-mediated CDC in chronic lymphoma cells.

However, the relevance of mCRP expression in breast cancer tissue with the prognosis of trastuzumab in a clinical setting remains unknown. In this study, we detected expression levels of CD55, CD59 and CD46 in formalin-fixed paraffin-embedded (FFPE) tumor specimens from HER2positive breast cancer patients treated with trastuzumab as postoperative adjuvant therapy by immunohistochemical staining. The relationship between mCRP expression and prognosis following postoperative adjuvant therapy containing trastuzumab was retrospectively analyzed. Previous studies reported that indomethacin (Indo), 4-hydroxy tamoxifen (Tam), levamisole (LMS), cisplatin (DDP) and sodium butyrate can downregulate mCRP expression (21,24-27). Here, we treated breast cancer cells with the aforementioned drugs and detected their inhibitory effect on mCRP expression and subsequently enhanced trastuzumab-mediated CDC.

\section{Materials and methods}

Patients and tumor specimens. Through search of the Breast Cancer Information Management System of West China Hospital, Sichuan University, a total of 65 breast cancer patients who had undergone radical mastectomy during the period from 2007 to 2010 were included in the present study. The inclusion criteria included: i) pathological diagnosis of breast cancer; ii) HER2-positive breast cancer [3+ by immunohistochemical analysis (IHC) or with gene amplification by fluorescence in situ hybridization (FISH)]; iii) availability of standard treatment containing trastuzumab at least for 1 year; and iv) $>3$ years of complete follow-up record upon radical mastectomy. Exclusion criteria were: i) diagnosis of metastatic breast cancer before treatment; and ii) administration of any preoperative treatment. We collected the clinical information of patients and performed telephone follow-up periodically through the Breast Cancer Information Management System (once every 6 months during the initial 1-5 years and once yearly after 5 years). The study met the recommendations of the 'Declaration of Helsinki' and was approved by the Ethics Committee of West China Hospital.

The 65 FFPE breast tumor tissues and 14 FFPE normal breast tissues from 65 breast cancer patients were retrospectively collected from the Pathology Department of West China Hospital. Disease-free survival (DFS) was defined as the time from radical mastectomy to tumor recurrence (28). Relapse rate was defined as the proportion of the patients with recurrent disease during the follow-up period.

Immunohistochemical staining. The streptavidin-biotin complex (SABC) method was used to detect the expression levels of CD55, CD59 and CD46 in the FFPE specimens. The specimens were heated using a microwave at low power for 5 min for antigen retrieval. Three percent $\mathrm{H}_{2} \mathrm{O}_{2}$ deionized water solution was used to block endogenous peroxidase. Anti-CD55 (clone SM3030P; Acris Co., Gemany; 1:200), anti-CD59 (clone p282; Abcam Co., USA; 1:100) or anti-CD46 (clone H-294; Santa Cruz Biotechnology Co., USA; 1:100) antibodies were added to the samples and incubated at $4{ }^{\circ} \mathrm{C}$ overnight. After PBS washes, the biotinylated secondary antibody (ZB-2010 or ZB-2020; Zhongshan Golden Bridge Co., China) was added. The samples were incubated for $30 \mathrm{~min}$, and subjected to PBS washes. Afterwards, the samples were incubated with streptavidin-biotin-peroxidase labeled tertiary antibody (ZB-2404; Zhongshan) for $30 \mathrm{~min}$. Color was developed using diaminobenzidine (DAB) chromogenic color development reagent. PBS instead of the primary antibody was used in the negative control group. Staining intensity of the tumor cells was classified as:,,-+++ and +++ (Fig. 1). At least 1,000 tumor cells were assessed and high CD55, CD59 or CD46 expression was defined as +++ expression of CD55, CD59 or CD46 in more than $1 \%$ of breast cancer cells. Otherwise, the breast cancer specimens were identified as having low CD55, CD59 or CD46 expression (29)

Cell culture. The ER-/HER2 ${ }^{+}$breast cancer cell line SK-Br-3 and the $\mathrm{ER}^{+} / \mathrm{HER} 2^{+} \mathrm{BT}-474$ cell line (The Cell Bank, Chinese Academy of Sciences) $(30,31)$, were cultured in RPMI-1640 containing 10\% fetal bovine serum (FBS; Gibco, USA) and MEM (Gibco) containing 10\% FBS, respectively.

Cell cytotoxicity as determined by MTT assay. SK-Br-3 or BT-474 cells (1x10 ${ }^{4}$ cells/well) were seeded in 96-well plates and incubated overnight to allow cells to adhere. The cells were pretreated with the following drugs: Indo $(20,40,80,160$ and $320 \mu \mathrm{M})$, Tam $(1,2,4,8$ and $16 \mu \mathrm{M})$, LMS $(0.16,0.32$, $0.64,1.28$ and $2.56 \mathrm{mM})$, sodium butyrate $(0.3,0.6,1.2,2.4$ and $4.8 \mathrm{mM}$ ) (all from Sigma USA) or DDP $(0.25,0.5,1,2$ and $4 \mu \mathrm{M}$; Shandong Qilu Pharmaceutical Factory, China) for 72 h. 3-(4,5-Dimethylthiazol-2-yl)-2,5-diphenyltetrazolium bromide (MTT; Sigma) was then added and incubated for 
$4 \mathrm{~h}$. Absorbance at $490 \mathrm{~nm}$ was measured using a Multiskan Spectrum (Thermo Scientific Co., USA). The maximum non-toxic doses of the drugs in the SK-Br-3 or BT-474 cells were determined.

Detection of trastuzumab-mediated CDC effects. The SK-Br-3 and BT-474 cell lines were used to study trastuzumab-induced CDC. Normal human serum (NHS) obtained from healthy volunteers (Blood Bank of West China Hospital, Sichuan University, China) was provided voluntarily for this study. Cells were incubated with heat-inactivated NHS $\left(56^{\circ} \mathrm{C}\right.$, $60 \mathrm{~min}$ ) as the negative control. Phosphatidylinositol-specific phospholipase C (PI-PLC; Invitrogen Co., USA) specifically hydrolyzes and inactivates anchor proteins CD55 and CD59 (32). Cells were incubated with PI-PLC as the positive control. Breast cancer cells were pretreated with maximum non-toxic doses of Indo $(40 \mu \mathrm{M})$, Tam $(2 \mu \mathrm{M}), \mathrm{LMS}(0.16 \mathrm{mM})$, sodium butyrate $(0.5 \mu \mathrm{M})$ and DDP $(0.32 \mathrm{mM})$ for $72 \mathrm{~h}$, and subsequently washed with PBS. Cells were detached by $0.125 \%$ trypsin and washed with PBS. A total of $1 \times 10^{5}$ cells were then cultured with FBS-free medium containing 50\% human serum (v/v) and $50 \mu \mathrm{g} / \mathrm{ml}$ trastuzumab for $1 \mathrm{~h}$ (32). Afterwards, trypan blue staining was performed to count the dead and viable cell number in a hemocytometer under a microscope. Experiments were repeated at least three times. The cell death rate was expressed as the percentage of dead cells relative to the total number of cells (viable cells and dead cells included).

Detection of CD55 mRNA expression levels using $q R T-P C R$. Cells were pretreated with $2 \mu \mathrm{M}$ Tam for 6,12 and $24 \mathrm{~h}$. Total cellular RNA was extracted using the BIOzol total RNA extraction kit (BioFlux Co., Japan) according to the manufacturer's instructions. First-strand cDNAs were synthesized from total RNA using M-MLV transcriptase and oligo-dT primer. Quantitative RT-PCR (qRT-PCR) was performed using duplicate SsoFast ${ }^{\text {TM }}$ Eva Green ${ }^{\circledR}$ Supermix kit (Bio-Rad, USA) in Chromo4 real-time PCR detector (Bio-Rad) following the manufacturer's instructions. The primer sequences used are presented in Table I (33). The relative quantitation of gene expression was performed using the comparative $\mathrm{Ct}(\Delta \Delta \mathrm{Ct})$ method with GAPDH as a housekeeping gene. Experiments were repeated at least twice.

Western blot assays. After pretreatment with $2 \mu \mathrm{M}$ (maximum non-toxic doses) Tam for $72 \mathrm{~h}, \mathrm{SK}-\mathrm{Br}-3$ and BT-474 cells were lysed at $4^{\circ} \mathrm{C}$ in RIPA lysis buffer (Biyuntian Co., China). Total protein concentration was determined using the Bradford protein assay (Bio-Rad). Equal amounts of cell lysates were separated on $12 \%$ SDS-PAGE and transferred to PVDF membranes. PVDF membranes were blocked with $5 \%$ nonfat milk in PBS-T (0.5\% of Tween-20 in PBS). The membranes were incubated at $4{ }^{\circ} \mathrm{C}$ overnight with the primary antibodies against CD55 (BRIC216), CD59 (YTH53.1) or $\beta$-actin (all from Santa Cruz Biotechnology, USA). After PBS-T washing, horseradish peroxidase-conjugated secondary antibodies (Santa Cruz Biotechnogy) were added. The membranes were then incubated at room temperature for $1 \mathrm{~h}$. Individual protein bands were visualized using chemiluminescence reagent (Millipore, USA). The film was exposed in a dark room and
Table I. Primer sequences.

Sequence

detected

Primer sequence

\begin{tabular}{ll}
\hline CD59 & F: 5'-TAACCCAACTGCTGACTGCAA-3' \\
& R: 5'-TTTGGTAATGAGACACGCATCAA-3' \\
CD55 & F: 5'-TTCCCCCAGATGTACCTAATGC-3' \\
& R: 5'-TTACAGTATCCTCGGGAAAACTTGT-3' \\
GAPDH & F: 5'-GAAGGTGAAGGTCGGAGTC-3' \\
& R: 5' -GAAGATGGTGATGGGATTTC-3' \\
\hline
\end{tabular}

F, forward; R, reverse.

scanned with HP LaserJet 3055 scanner. Volume densitometry of the immunoreactive bands was quantitated with One Quantity software (Bio-Rad, USA).

Detection of $m C R P$ expression levels by flow cytometry. SK-Br-3 and BT-474 cells were pretreated with the maximum non-toxic doses of Indo, Tam, LMS, DDP or sodium butyrate for $72 \mathrm{~h}$ and washed with PBS. Cells were incubated on ice for 30 min with the FITC-conjugated anti-CD55 antibody, FITC-conjugated anti-CD59 antibody (Beijing Jingmei Gene Valley Technology Co., China) or FITC-conjugated anti-CD46 antibody (Biolegend Co., USA). IgG1 isotype control/FITC (Beijing Jingmei Gene Valley Technology Co., China) was used as the negative control antibody. Then, the cells were washed, and resuspended in formaldehyde fixing solution $(1 \%$ BSA-PBS $+1 \%$ polyphosphate formaldehyde), and subjected to flow cytometry (FCM).

Statistical analysis. Statistical significance of the correlation between the clinicopathologic characteristics or prognosis and the patients with mCRP high expression and low expression was determined with a $\chi^{2}$ test comparison. Kaplan-Meier survival analysis was used for estimation of DFS, and log-rank test was used for inter-group comparison. Cox proportional hazards model analysis was used for estimation of univariate hazard ratios (HRs) for DFS and multivariate analysis. Menopausal status, tumor size $(\mathrm{cm})$, tumor grade, stage, lymph node metastasis status, hormone receptor status and mCRP expression were included in the model. The CDC of trastuzumab was expressed as mean \pm SEM. Independent samples t-test was used for comparison between two groups. $\mathrm{P}<0.05$ was considered to indicate a statistically significant difference. SPSS software version 16.0 (SPSS Inc., USA) was used for the statistical analysis.

\section{Results}

Clinicopathological characteristics of the patients. The clinicopathological characteristics of the 65 breast cancer patients who underwent postoperative adjuvant treatment containing trastuzumab are summarized in Table II. Patient age ranged from 21 to 67 years (with a median age of 49 years). Adjuvant chemotherapy plus trastuzumab was administered to 43 
Table II. Patient characteristics.

\begin{tabular}{|c|c|}
\hline Characteristics & $\begin{array}{l}\text { No. of patients } \\
\qquad(\mathrm{n}=65)\end{array}$ \\
\hline \multicolumn{2}{|l|}{ Age (years) } \\
\hline Median & 49 \\
\hline Range & $21-67$ \\
\hline \multicolumn{2}{|l|}{ Menopausal status } \\
\hline Premenopausal & 27 \\
\hline Postmenopausal & 38 \\
\hline \multicolumn{2}{|l|}{ Histologic type } \\
\hline Invasive ductal carcinoma & 65 \\
\hline \multicolumn{2}{|l|}{ Tumor size (cm) } \\
\hline$\leq 2$ & 28 \\
\hline$>2-<5$ & 31 \\
\hline$\geq 5$ & 6 \\
\hline \multicolumn{2}{|l|}{ Histologic grade } \\
\hline $\mathrm{I} / \mathrm{II}$ & 15 \\
\hline III & 50 \\
\hline \multicolumn{2}{|l|}{ Tumor stage } \\
\hline I & 14 \\
\hline II & 31 \\
\hline III & 20 \\
\hline \multicolumn{2}{|l|}{ Lymph node metastasis } \\
\hline Positive & 37 \\
\hline Negative & 28 \\
\hline \multicolumn{2}{|l|}{ Estrogen receptor status } \\
\hline Positive & 20 \\
\hline Negative & 45 \\
\hline \multicolumn{2}{|l|}{ Progesterone receptor status } \\
\hline Positive & 20 \\
\hline Negative & 45 \\
\hline \multicolumn{2}{|l|}{ Prognosis } \\
\hline Recurrence & 13 \\
\hline Non-recurrence & 52 \\
\hline \multicolumn{2}{|l|}{ Adjuvant therapy } \\
\hline Chemotherapy & 43 \\
\hline Chemohormonotherapy & 22 \\
\hline
\end{tabular}

patients and adjuvant endocrine therapy plus adjuvant chemotherapy and trastuzumab to 22 patients. Trastuzumab was given once every three weeks with a starting dose of $8 \mathrm{mg} / \mathrm{kg}$ and a maintenance dose of $6 \mathrm{mg} / \mathrm{kg}$, following chemotherapy. Trastuzumab therapy was maintained for at least 1 year. The median follow-up duration was 47 months (range from 24 to 75 months). Relapse occurred in 13 patients during the followup period. Two patients relapsed within 1 year, 10 patients within 1-3 years, and 1 patient within 3-5 years. Among them, 5 patients had lymph node metastasis, 4 patients had lung metastasis, 2 patients had bone metastasis, and 2 patients had gastrointestinal metastasis.

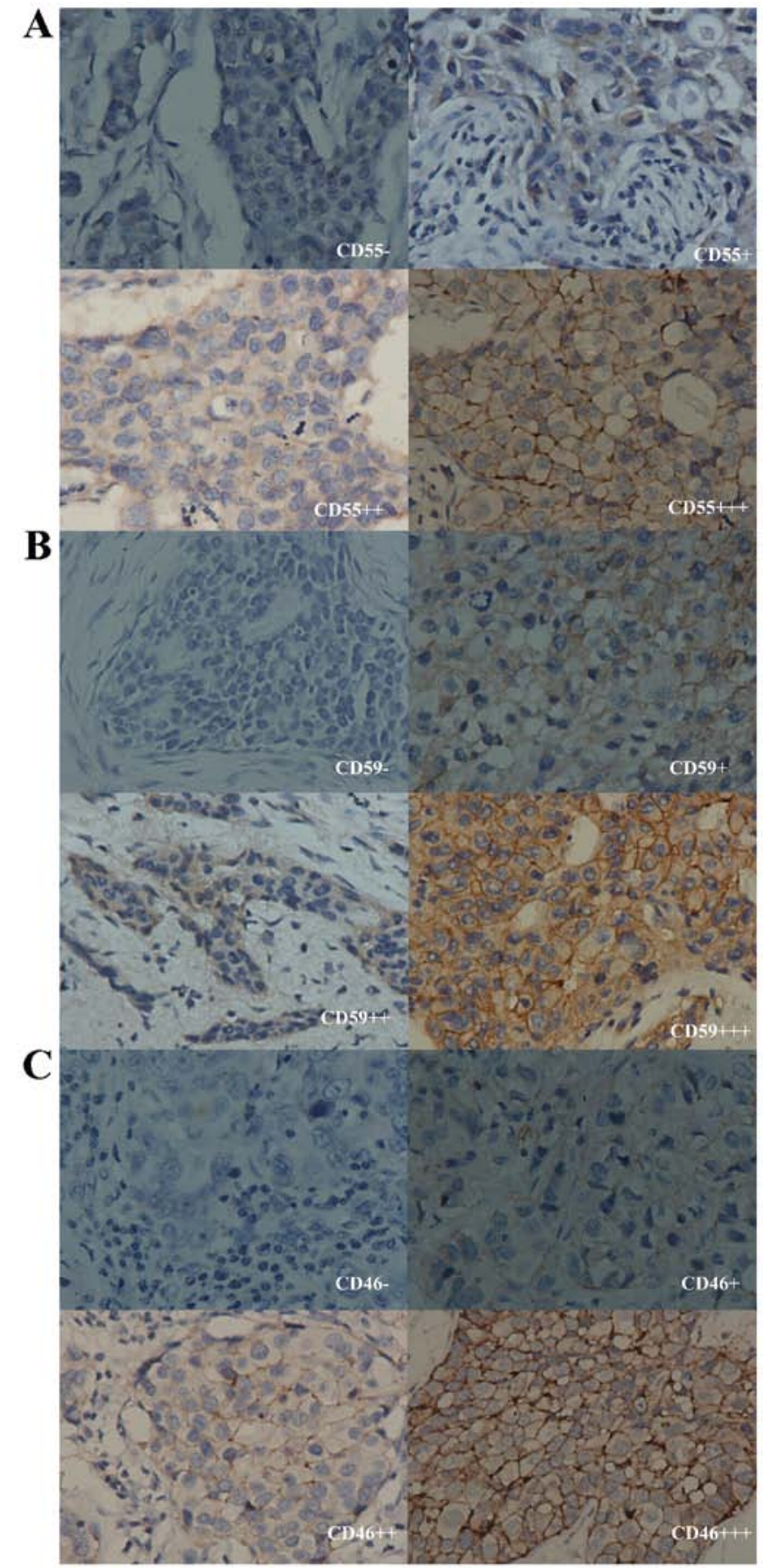

Figure 1. Expression levels of mCRPs (CD55, CD59 and CD46) in the breast cancer samples (SP, $\mathrm{x} 400)$ as detected by immunohistochemistry. The expression of (A) CD55, (B) CD59 and (C) CD46 in breast cancer samples.

Expression levels of $m C R P s$ in the breast tumors are associated with the relapse rate. Levels of CD55, CD59 and CD46 expression in the FFPE breast tumor tissues from 65 patients who underwent postoperative adjuvant therapy containing trastuzumab were detected by immunohistochemistry. CD55, CD59 and CD46 were expressed on the membrane of the breast cancer cells (Fig. 1). Among the patients, 30/65 (46.2\%) had high expression levels of CD55, 29 (44.6\%) had a high expression level of CD59, and 29 (44.6\%) had a high expression level of CD46. In comparison, only 2/14 (14.3\%) normal breast tissue specimens had high expression levels of CD55, 
Table III. Correlation between CD55, CD59 and CD46 expression and clinicopathological variables of the breast cancer patients.

\begin{tabular}{|c|c|c|c|c|c|c|c|c|c|c|}
\hline \multirow[b]{2}{*}{ Characteristics } & \multirow[b]{2}{*}{$\%$} & \multicolumn{3}{|c|}{ CD55 } & \multicolumn{3}{|c|}{ CD59 } & \multicolumn{3}{|c|}{ CD46 } \\
\hline & & $\begin{array}{c}\text { Low } \\
(53.8 \%)\end{array}$ & $\begin{array}{c}\text { High } \\
(46.2 \%)\end{array}$ & P-value & $\begin{array}{l}\text { Low } \\
(55.4 \%)\end{array}$ & $\begin{array}{l}\text { High } \\
(44.6 \%)\end{array}$ & P-value & $\begin{array}{c}\text { Low } \\
(55.4 \%)\end{array}$ & $\begin{array}{l}\text { High } \\
(44.6 \%)\end{array}$ & P-value \\
\hline Menopausal status & & & & 0.437 & & & 0.135 & & & 0.981 \\
\hline Premenopausal & 41.5 & 13 & 14 & & 12 & 15 & & 15 & 12 & \\
\hline Postmenopausal & 58.5 & 22 & 16 & & 24 & 14 & & 21 & 17 & \\
\hline Tumor size $(\mathrm{cm})$ & & & & 0.536 & & & 0.521 & & & 0.324 \\
\hline$\leq 2$ & 43.1 & 15 & 13 & & 16 & 12 & & 18 & 10 & \\
\hline$>2-<5$ & 47.7 & 18 & 13 & & 18 & 13 & & 16 & 15 & \\
\hline$\geq 5$ & 9.2 & 2 & 4 & & 2 & 4 & & 2 & 4 & \\
\hline Histologic grade & & & & 0.525 & & & 0.682 & & & 0.682 \\
\hline $\mathrm{I} / \mathrm{II}$ & 23.1 & 7 & 8 & & 9 & 6 & & 9 & 6 & \\
\hline III & 76.9 & 28 & 22 & & 27 & 23 & & 27 & 23 & \\
\hline Stage & & & & 0.317 & & & 0.246 & & & 0.709 \\
\hline I & 21.5 & 8 & 6 & & 9 & 5 & & 9 & 5 & \\
\hline II & 47.7 & 19 & 12 & & 19 & 12 & & 17 & 14 & \\
\hline III & 30.8 & 8 & 12 & & 8 & 12 & & 10 & 10 & \\
\hline Lymph node metastasis & & & & 0.643 & & & 0.078 & & & 0.209 \\
\hline Positive & 56.9 & 19 & 18 & & 17 & 20 & & 18 & 19 & \\
\hline Negative & 43.1 & 16 & 12 & & 19 & 9 & & 18 & 10 & \\
\hline Estrogen receptor status & & & & 0.368 & & & 0.465 & & & 0.072 \\
\hline Positive & 30.8 & 12 & 8 & & 13 & 8 & & 15 & 6 & \\
\hline Negative & 69.2 & 23 & 22 & & 23 & 21 & & 21 & 23 & \\
\hline Progesterone receptor status & & & & 0.507 & & & 0.298 & & & 0.114 \\
\hline Positive & 30.8 & 12 & 8 & & 13 & 7 & & 14 & 6 & \\
\hline Negative & 69.2 & 23 & 22 & & 23 & 22 & & 22 & 23 & \\
\hline Prognosis & & & & 0.013 & & & 0.046 & & & 0.454 \\
\hline Recurrence & 20.0 & 3 & 10 & & 4 & 9 & & 6 & 7 & \\
\hline Non-recurrence & 80.0 & 32 & 20 & & 32 & 20 & & 30 & 22 & \\
\hline Adjuvant therapy & & & & 0.544 & & & 0.667 & & & 0.338 \\
\hline Chemotherapy & 66.2 & 22 & 21 & & 23 & 20 & & 22 & 21 & \\
\hline Chemohormonotherapy & 33.8 & 13 & 9 & & 13 & 9 & & 14 & 8 & \\
\hline
\end{tabular}

CD59 or CD46; the remaining 12 (85.7\%) specimens had low expression levels.

As shown in Table III, patients with CD55 or CD59 overexpression had significantly higher relapse rates than the patients with low expression of CD55 (33.3 vs. 8.6\%; $\mathrm{P}=0.013$ ) or CD59 (31.0 vs. 11.1\%; $\mathrm{P}=0.046$ ). The relapse rate in the CD46 overexpression group was slightly higher than that in the CD46 low expression group (24.1 vs. 16.7\%). However, the difference was not statistically significant $(\mathrm{P}=0.454)$. Other clinicopathological characteristics, including menopausal status, histological classification, tumor stage, lymph node metastasis, and hormone receptor status did not differ significantly between patients with high and low expression of mCRPs $(\mathrm{P}>0.05)$.

Expression levels of $m C R P s$ in breast cancer are associated with prognosis. Mean DFS of 65 patients included in our study was 64 months (95\% CI, 59-69 months). We found that the mean DFS of patients with CD55 or CD59 high expression was significantly shorter when compared to the patients with low expression of CD55 (56 vs. 70 months; log-rank test, $\mathrm{P}=0.008$ ) or $\mathrm{CD} 59$ (56 vs. 69 months; log-rank test, $\mathrm{P}=0.033$ ). In contrast, the mean DFS was not significantly different between patients with high and low expression of CD46 (66 vs. 60 months; log-rank test, $\mathrm{P}=0.516$; Fig. 2). Risk factors of DFS including menopausal status, tumor size $(\mathrm{cm})$, tumor grade and stage, lymph node metastasis, hormone receptor status and mCRP expression were analyzed using Cox proportional hazards model. Univariate analysis revealed that overexpression of CD55 (HR=4.822; 95\% CI, 1.323-17.57; $\mathrm{P}=0.02)$ or CD59 (HR=3.343; 95\% CI, 1.027-10.878; $\mathrm{P}=0.05)$, but not CD46 (HR=1.43; 95\% CI, 0.48-4.257; $\mathrm{P}=0.52$ ), was associated with a high risk of relapse. Multivariate analysis showed that CD55 overexpression was an independent risk factor of relapse 
Table IV. Univariate and multivariate analysis of the prognostic factors.

\begin{tabular}{llcr}
\hline & \multicolumn{1}{c}{ Univariate } & & Multivariate \\
\cline { 2 - 3 } & \multicolumn{1}{c}{ HR $(95 \% \mathrm{CI})$} & P-value & HR (95\% CI) \\
\hline Menopausal status & $0.497(0.166-1.485)$ & 0.21 & \\
Tumor size (cm) & $1.032(0.787-1.352)$ & 0.82 & \\
Histologic grade & $0.968(0.266-3.518)$ & 0.96 & \\
Stage & $1.173(0.531-2.59)$ & 0.69 & \\
Lymph node metastasis & $1.291(0.422-3.947)$ & 0.66 & \\
Estrogen receptor status & $0.732(0.239-2.239)$ & 0.59 & \\
Progesterone receptor status & $0.610(0.199-1.866)$ & 0.39 & 0.25 \\
CD59 & $3.343(1.027-10.878)$ & 0.05 & $0.543-10.414)$ \\
CD55 & $4.822(1.323-17.570)$ & 0.02 & $0.985-22.974)$ \\
CD46 & $1.430(0.48-4.257)$ & 0.52 & \\
\hline
\end{tabular}
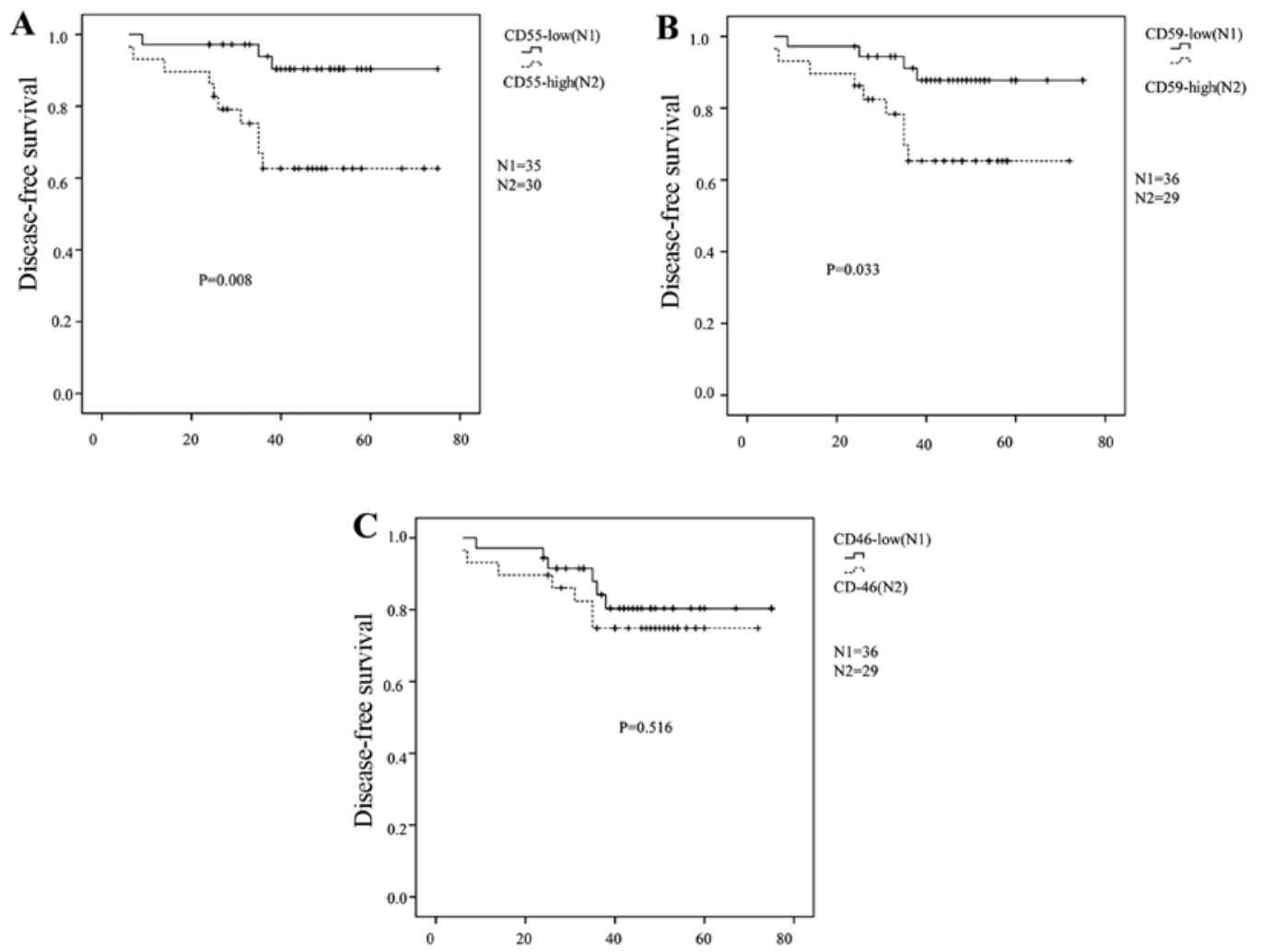

Figure 2. Disease-free survival (DFS) curves of breast cancer patients with high and low expression levels of mCRPs. (A and B) A statistically significant difference was observed in DFS between the (A) CD55-low expression and the CD55-high expression group $(\mathrm{P}<0.05)$ and the (B) CD59-low expression and CD59-high expression group $(\mathrm{P}<0.05)$. (C) No statistically significant difference $(\mathrm{P}=0.516)$ was observed between the $\mathrm{CD} 46$-low expression and $\mathrm{CD} 46-\mathrm{high}$ expression group in regard to DFS $(\mathrm{P}>0.05)$.

$(\mathrm{HR}=4.757 ; 95 \%$ CI, 0.985-22.974; $\mathrm{P}=0.05)$. However, CD59 overexpression was not significantly associated with risk of relapse (HR=2.378; 95\% CI, 0.543-10.414; $\mathrm{P}=0.25)$ (Table IV).

Inhibition of $m$ CRP expression enhances trastuzumab-mediated $C D C$. Our retrospective analysis of clinical samples revealed that $\mathrm{CD} 55$ overexpression is an independent risk factor of relapse in breast cancer patients treated with trastuzumab. It is possible that a high CD55 expression level suppresses trastuzumab-mediated CDC, and thus leads to poor prognosis. Therefore, we aimed to ascertain whether downregulation of mCRP expression could sensitize breast cancer cells to trastuzumab-mediated CDC. We confirmed the maximum non-toxic doses of Indo, Tam, LMS, DDP and sodium butyrate (40, 2, $0.16,0.5$ and $0.32 \mathrm{mM}$, respectively) in SK-Br3 and BT-474 cells by MTT. Breast cancer cells were pretreated with these drugs at their maximum non-toxic doses. Flow cytometric results showed that only Tam inhibited expression levels of 

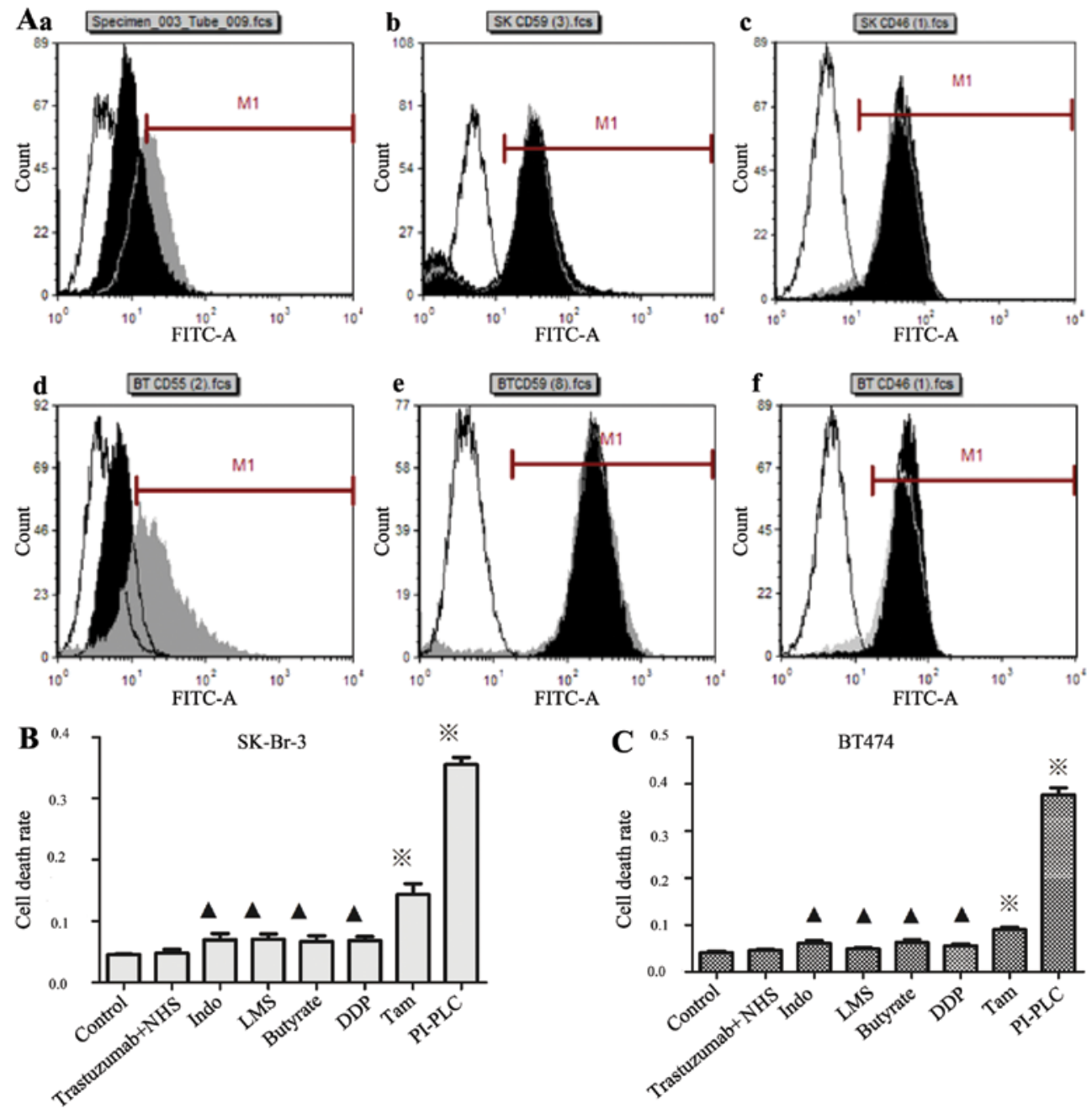

Figure 3. Effects of the different drug treatments on mCRP expression and trastuzumab-induced CDC in SK-Br-3 and BT-474 cells. (A) (a-c) Representative FACS histograms show the expression levels of CD55, CD59 and CD46 in the SK-Br-3 cell line after Tam treatment. (d-f) Representative FACS histograms show the expression of CD55, CD59 and CD46 in the BT-474 cell line after Tam treatment. (B and C) Compared with the Trastuzumab and NHS group, no statistically significant difference was noted; ${ }^{\wedge} \mathrm{P}>0.05$. Significantly different from the trastuzumab and NHS group; ${ }^{*} \mathrm{P}<0.05$. NHS, normal human serum; Indo, indomethacin; LMS, levamisole; DDP, cisplatin; Tam, 4-hydroxy tamoxifen; PI-PLC, phosphatidylinositol-specific phospholipase C.
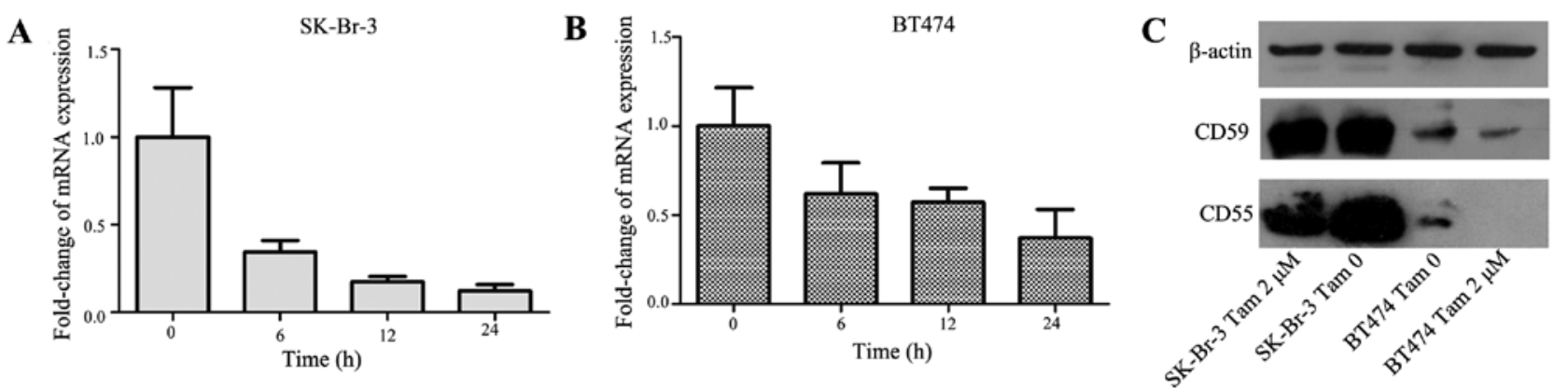

Figure 4. mRNA and protein expression levels of CD55 as detected by quantitative RT-PCR and western blot analysis. (A and B) mRNA levels of CD55 in SK-Br-3 and BT-474 cells after pretreatment with Tam for 6, 12 and $24 \mathrm{~h}$. mRNA levels of CD55 were calculated using n-fold change of mRNA (relative to the control). (C) Following pretreatment of SK-Br-3 and BT-474 cells with Tam for $72 \mathrm{~h}$, western blotting was performed to detect the expression of CD55 and CD59. Steady-state $\beta$-actin protein levels served as the protein loading control.

CD55 in the SK-BR-3 and BT-474 cells (Fig. 3A). Real-time PCR and western blotting results confirmed the inhibitory effect of Tam on both the mRNA and protein expression levels of CD55 (Fig. 4). In contrast, CD59 expression levels in both breast cancer cell lines did not change significantly after Tam pretreatment.

To investigate the effect of mCRP inhibition of trastuzumab-mediated CDC, we pretreated breast cancer cells with 
Tam, DDP, Indo, LMS or sodium butyrate for $72 \mathrm{~h}$, and then incubated the cells with 50\% normal human serum (NHS) and trastuzumab for $1 \mathrm{~h}$. Trastuzumab with NHS induced an insignificant cytotoxic response $(4.8 \pm 0.5$ vs. $4.6 \pm 0.3 \%$, respectively in the SK-Br3 and BT-474 cells), compared with the negative control group $(4.5 \pm 0.01$ vs. $4.1 \pm 0.04 \%$, respectively), while Tam pretreatment significantly enhanced the cell death rate induced by trastuzumab and NHS in the SK-Br3 $(14.33 \pm 1.7 \%$, $\mathrm{P}<0.01)$ and $\mathrm{BT}-474$ cells $(9.0 \pm 0.6 \%, \mathrm{P}<0.01)$ (Fig. 3B and $\mathrm{C})$. In contrast, DDP, Indo, LMS, or sodium butyrate pretreatment did not significantly affect trastuzumab-mediated CDC (P>0.05; Fig. 3B and C).

\section{Discussion}

Overexpression of mCRPs has been implicated in malignant tumors and is associated with poor prognosis (17-21,34-36). Durrant et al (34) reported that CD55 is highly expressed in colorectal cancer and is an indicator of poor prognosis in colorectal cancer patients. Madjd et al (36) showed that high expression levels of CD46 in breast cancer tissues were positively correlated with the degree of tumor malignancy, poor prognosis and risk of recurrence. Moreover, two studies reported that $\mathrm{mCRP}$ overexpression was related to tolerance of lymphoma to rituximab-mediated CDC $(22,37)$. Bannerji et al (38) reported that rituximab-induced CDC was enhanced significantly by reducing CD59 and CD55 expression in leukocytes from patients with chronic lymphocytic leukemia.

In the present study, we assessed the expression levels of CD55, CD59 and CD46 in 65 FFPE breast tumor tissues and in 14 FFPE normal breast tissues from 65 HER2-positive breast cancer patients who underwent trastuzumab treatment. Our data demonstrated that breast cancer tissues had higher expression of CD55, CD59 and CD46 than that in the normal tissues. Furthermore, subgroup analysis revealed that high expression levels of CD55 and CD59 were associated with higher recurrence rate, and CD55 overexpression was found to be an independent risk factor of recurrence, suggesting that CD55 overexpression may be an indicator of poor prognosis of operable breast cancer treated with adjuvant trastuzumab. In contrast CD55 expression was not associated with other clinicopathological features, including tumor stage, histological grade, or hormone receptor status in breast cancer. This is consistent with studies by Durrant et al (34) and Ikeda et al (29). This is the first study, to our knowledge, to investigate the predictive role of the expression of three mCRPs (CD55, CD59, and CD46) in the prognosis of breast cancer patients who underwent postoperative adjuvant therapy containing trastuzumab.

Emerging evidence has shown that neutralizing antibodies or siRNAs that downregulate mCRPs can significantly enhance mAb-mediated CDC in vitro $(22,23,37)$. Bellone et al (39) found that trastuzumab-induced CDC was enhanced after silencing of CD55 and CD59 by siRNAs in uterine cancer cells. Zhao et al (21) drew similar conclusions in two non-small cell lung cancer cell lines, A549 and H157. Mamidi et al (10) reported that silencing of membrane regulators (CD46, CD55 and CD59) by anti-mCRP siRNAs enhanced the CDC of trastuzumab and pertuzumab. However, mCRPs are widely expressed throughout the human body, thus neutralizing antibodies and siRNAs of mCRPs may damage normal tissues. Currently, there are no effective neutralizing antibodies to mCRPs that can be applied in clinical practice. Researchers have attempted to solve this challenge by bi-specific antibodies, i.e. engineered antibodies that target tumor cells and mCRPs simultaneously (40). However, specificity remains to be the bottleneck of this therapeutic strategy.

In the present study, we treated breast cancer cells with commonly used clinical drugs that have been reported to reduce mCRP expression levels. We expected that these drugs would effectively reduce mCRP expression levels without increasing toxicity, and improve the efficacy of trastuzumab. We found that 4-hydroxy tamoxifen (Tam) effectively downregulated CD55 expression, but not CD59 and CD46. Pretreatment of Tam enhanced the trastuzumab-mediated CDC in BT-474 and SK-Br3 cells. Several in vitro studies have shown that combined use of Tam and trastuzumab can synergistically inhibit the growth of breast cancer cells $(41,42)$. The authors suggested that suppression of ER and HER-2/neu pathways simultaneously in $\mathrm{HER}^{+} / \mathrm{ER}^{+}$breast cancer may improve the efficacy of breast cancer therapy. Our results suggest that Tam and trastuzumab can synergistically kill breast cancer cells by enhancing trastuzumab-mediated CDC.

In the present study, we found that Tam could not only reduce CD55 expression in $\mathrm{HER} 2^{+} / \mathrm{ER}^{+} \mathrm{BT}-474$ cells but also in HER $2^{+} / \mathrm{ER}^{-} \mathrm{SK}-\mathrm{Br} 3$ cells. These data raise the possibility that combined Tam and trastuzumab treatment may also enhance the efficacy of trastuzumab in patients with ER-negative breast cancer, besides ER-positive breast cancer. The efficacy and feasibility of combined Tam and trastuzumab for treatment of breast cancer warrants further investigation. In addition, the mechanism of Tam-mediated downregulation of CD55 in SK-Br3 and BT-474 cells remains unclear. CD55 expression is possibly downregulated by modulation of certain cytokines, such as interferon (IFN) (41-43). Our results showed that both CD55 mRNA and protein levels were simultaneously reduced by Tam treatment, suggesting that regulation of CD55 expression could occur at the transcriptional level.

In conclusion, high expression levels of CD55 and CD59 were associated with a higher risk of recurrence, and CD55 overexpression was an independent risk factor of recurrence in breast cancer patients who received postoperative adjuvant therapy containing trastuzumab. In addition, trastuzumabinduced CDC was enhanced in vitro by Tam treatment through CD55 downregulation. These results warrant further investigation in regards to the predictive role of CD55 and CD59 in the efficacy of trastuzumab in postoperative adjuvant therapy of breast cancer. Furthermore, combined use of Tam and trastuzumab may enhance trastuzumab-induced CDC, and thus potentially improve the efficacy of trastuzumab, which requires additional clinical studies to verify.

\section{Acknowledgements}

This study was supported by the National Natural Sciences Foundation of China (30972940), West China Hospital and Shanghai Roche Pharmaceuticals Ltd., horizontal cooperation project (H1109114 and H1307060). We would like to thank Shanghai Roche Pharmaceuticals Ltd., for the editorial assistance. 


\section{References}

1. Natsume A, Niwa R and Satoh M: Improving effector functions of antibodies for cancer treatment: Enhancing ADCC and CDC. Drug Des Devel Ther 3: 7-16, 2009.

2. Yamashita M,Katakura Y and Shirahata S: Recent advances in the generation of human monoclonal antibody. Cytotechnology 55: 55-60, 2007.

3. Piccart-Gebhart MJ, Procter M, Leyland-Jones B, et al: Trastuzumab after adjuvant chemotherapy in HER2-positive breast cancer. N Engl J Med 353: 1659-1672, 2005.

4. Romond EH, Perez EA, Bryant J, et al: Trastuzumab plus adjuvant chemotherapy for operable HER2-positive breast cancer. N Engl J Med 353: 1673-1684, 2005.

5. Slamon D, Eiermann W, Robert N, et al: Phase III randomized trial comparing doxorubicin and cyclophosphamide followed by docetaxel $(\mathrm{AC} \rightarrow \mathrm{T}$ ) with doxorubicin and cyclophosphamide followed by docetaxel and trastuzumab $(\mathrm{AC} \rightarrow \mathrm{TH})$ with docetaxel, carboplatin and trastuzumab (TCH) in HER2 positive early breast cancer patients: study. Breast Cancer Res Treat 94: S5 2005.

6. Nahta R and Esteva FJ: HER2 therapy: molecular mechanisms of trastuzumab resistance. Breast Cancer Res 8: 215, 2006.

7. Valabrega G, Montemurro F and Aglietta M: Trastuzumab: mechanism of action, resistance and future perspectives in HER2-overexpressing breast cancer. Ann Oncol 18: 977-984, 2007.

8. Dalle S, Thieblemont C, Thomas L and Dumontet C: Monoclonal antibodies in clinical oncology. Anticancer Agents Med Chem 8: 523-532, 2008

9. Drebin JA, Link VC and Greene MI: Monoclonal antibodies specific for the neu oncogene product directly mediate antitumor effects in vivo. Oncogene 2: 387-394, 1988.

10. Mamidi S, Cinci M, Hasmann M, Fehring V and Kirschfink M: Lipoplex mediated silencing of membrane regulators (CD46, CD55 and CD59) enhances complement-dependent antitumor activity of trastuzumab and pertuzumab. Mol Oncol 7: 580-594 2013.

11. Prang N, Preithner S, Brischwein K, et al: Cellular and complement-dependent cytotoxicity of Ep-CAM-specific monoclona antibody MT201 against breast cancer cell lines. Br J Cancer 92: 342-349, 2005.

12. Yu J, Caragine T, Chen S, Morgan BP, Frey AB and Tomlinson $S$ : Protection of human breast cancer cells from complement-mediated lysis by expression of heterologous CD59. Clin Exp Immunol 115: 13-18, 1999.

13. Mineo JF, Bordron A, Quintin-Roue I, et al: Recombinant humanised anti-HER2/neu antibody (Herceptin) induces cellular death of glioblastomas. Br J Cancer 91: 1195-1199, 2004.

14. Fishelson Z, Donin N, Zell S, Schultz S and Kirschfink M: Obstacles to cancer immunotherapy: expression of membrane complement regulatory proteins (mCRPs) in tumors. Mol Immunol 40: 109-123, 2003.

15. Gancz D and Fishelson Z: Cancer resistance to complementdependent cytotoxicity (CDC): Problem-oriented research and development. Mol Immunol 46: 2794-2800, 2009.

16. Macor P and Tedesco F: Complement as effector system in cancer immunotherapy. Immunol Lett 111: 6-13, 2007.

17. Hosch SB, Scheunemann P, Lüth M, et al: Expression of 17-1A antigen and complement resistance factors CD55 and CD59 on liver metastasis in colorectal cancer. J Gastrointest Surg 5: 673-679, 2001

18. Koretz K, Brüderlein S, Henne C and Möller P: Decayaccelerating factor (DAF, CD55) in normal colorectal mucosa, adenomas and carcinomas. Br J Cancer 66: 810-814, 1992.

19. Liu YC, Chen L, Peng SY and Hoang-Vu C: Role of CD97 (stalk) and CD55 as molecular markers for prognosis and therapy of gastric carcinoma patients. J Zhejiang Univ Sci 6: 913-918, 2005.

20. Loberg RD, Day LL, Dunn R, Kalikin LM and Pienta KJ: Inhibition of decay-accelerating factor (CD55) attenuates prostate cancer growth and survival in vivo. Neoplasia 8: 69-78, 2006.

21. Zhao WP, Zhu B, Duan YZ and Chen ZT: Neutralization of complement regulatory proteins CD55 and CD59 augments therapeutic effect of herceptin against lung carcinoma cells Oncol Rep 21: 1405-1411, 2009.

22. Terui Y, Sakurai T, Mishima Y, et al: Blockade of bulky lymphoma-associated CD55 expression by RNA interference overcomes resistance to complement-dependent cytotoxicity with rituximab. Cancer Sci 97: 72-79, 2006.
23. Golay J, Lazzari M, Facchinetti V, et al: CD20 levels determine the in vitro susceptibility to rituximab and complement of B-cell chronic lymphocytic leukemia: further regulation by CD55 and CD59. Blood 98: 3383-3389, 2001.

24. Andoh A, Shimada M, Araki Y, Fujiyama Y and Bamba T: Sodium butyrate enhances complement-mediated cell injury via down-regulation of decay-accelerating factor expression in colonic cancer cells. Cancer Immunol Immunother 50: 663-672, 2002.

25. Holla VR, Wang D, Brown JR, Mann JR, Katkuri S and DuBois RN: Prostaglandin E2 regulates the complement inhibitor CD55/decay-accelerating factor in colorectal cancer. J Biol Chem 280: 476-483, 2005.

26. Xin YC, Gui SH and Ge SQ: Tamoxifen modulates the expression of complement inhibitory proteins on chorio-carcinoma cell line JAR. Shanghai J Immunol 1: 20-23, 2003.

27. Bjørge L and Matre R: Down-regulation of CD59 (protectin) expression on human colorectal adenocarcinoma cell lines by levamisole. Scand J Immunol 42: 512-516, 1995.

28. Eisenhauer EA, Therasse P, Bogaerts J, et al: New response evaluation criteria in solid tumours: revised RECIST guideline (version 1.1). Eur J Cancer 45: 228-247, 2009.

29. Ikeda J, Morii E, Liu Y, et al: Prognostic significance of CD55 expression in breast cancer. Clin Cancer Res 14: 4780-4786, 2008

30. Molina MA, Codony-Servat J, Albanell J, Rojo F, Arribas J and Baselga J: Trastuzumab (Herceptin), a humanized anti-Her2 receptor monoclonal antibody, inhibits basal and activated Her2 ectodomain cleavage in breast cancer cells. Cancer Res 61: 4744-4749, 2001

31. Subik K, Lee JF, Baxter L, et al: The expression patterns of ER, PR, HER2, CK5/6, EGFR, Ki-67 and AR by immunohistochemical analysis in breast cancer cell lines. Breast Cancer 4: $35-41,2010$

32. Spear GT, Lurain NS, Parker CJ, Ghassemi M, Payne GH and Saifuddin M: Host cell-derived complement control proteins CD55 and CD59 are incorporated into the virions of two unrelated enveloped viruses. Human T cell leukemia/lymphoma virus type I (HTLV-I) and human cytomegalovirus (HCMV). J Immunol 155: 4376-4381, 1995.

33. Kolev MV, Tediose T, Sivasankar B, et al: Upregulating CD59: a new strategy for protection of neurons from complement-mediated degeneration. Pharmacogenomics J 10: 12-19, 2010.

34. Durrant LG, Chapman MA, Buckley DJ, Spendlove I, Robins RA and Armitage NC: Enhanced expression of the complement regulatory protein CD55 predicts a poor prognosis in colorectal cancer patients. Cancer Immunol Immunother 52: 638-642, 2003.

35. Loberg RD, Wojno KJ, Day LL and Pienta KJ: Analysis of membrane-bound complement regulatory proteins in prostate cancer. Urology 66: 1321-1326, 2005.

36. Madjd Z, Durrant LG, Pinder SE, et al: Do poor-prognosis breast tumours express membrane cofactor proteins (CD46)? Cancer Immunol Immunother 54: 149-156, 2005

37. Macor P, Tripodo C, Zorzet $\mathrm{S}$, et al: In vivo targeting of human neutralizing antibodies against CD55 and CD59 to lymphoma cells increases the antitumor activity of rituximab. Cancer Res 67: 10556-10563, 2007.

38. Bannerji R, Kitada S, Flinn IW, et al: Apoptotic regulatory and complement-protecting protein expression in chronic lymphocytic leukemia: relationship to in vivo rituximab resistance. J Clin Oncol 21: 1466-1471, 2003.

39. Bellone S, Roque D, Cocco E, et al: Downregulation of membrane complement inhibitors CD55 and CD59 by siRNA sensitises uterine serous carcinoma overexpressing Her2/neu to complement and antibody-dependent cell cytotoxicity in vitro: implications for trastuzumab-based immunotherapy. $\mathrm{Br} \mathrm{J}$ Cancer 106: 1543-1550, 2012.

40. Gelderman KA, Kuppen PJ, Okada N, Fleuren GJ and Gorter A: Tumor-specific inhibition of membrane-bound complement regulatory protein Crry with bispecific monoclonal antibodies prevents tumor outgrowth in a rat colorectal cancer lung metastases model. Cancer Res 64: 4366-4372, 2004.

41. Argiris A, Wang CX, Whalen SG and DiGiovanna MP: Synergistic interactions between tamoxifen and trastuzumab (Herceptin). Clin Cancer Res 10: 1409-1420, 2004.

42. Wang CX, Koay DC, Edwards A, et al: In vitro and in vivo effects of combination of trastuzumab (Herceptin) and tamoxifen in breast cancer. Breast Cancer Res Treat 92: 251-263, 2005.

43. Schild-Hay LJ, Leil TA, Divi RL, Olivero OA, Weston A and Poirier MC: Tamoxifen induces expression of immune responserelated genes in cultured normal human mammary epithelial cells. Cancer Res 69: 1150-1155, 2009. 